\title{
Poder de Mercado na Indústria de Mineração de Pedra Britada da Região Metropolitana de São Paulo*
}

\section{Analysis of Crushed Stone Industry's Market Power in Metropolitan Region of São Paulo}

\author{
Dênis Antônio Cunha** \\ Lucas Campio Pinha*** \\ Marcelo José Braga* *** \\ Alexandre Bragança Coelho****
}

\begin{abstract}
Resumo: O presente estudo tem como principal objetivo analisar a conduta das empresas que compõem a indústria mineradora de pedra britada da Região Metropolitana de São Paulo (RMSP) com base na Nova Organização Industrial Empírica (New Empirical Industrial Organization - NEIO). Essa conduta se refere ao exercício do poder de mercado e à formação de cartel após essas práticas terem sido identificadas e condenadas. Os resultados permitiram verificar que a indústria apresenta um pequeno, mas significativo, poder de mercado. Ao mesmo tempo, o comportamento da indústria está distante de um cartel. Dessa forma, conclui-se que as denúncias e as punições aplicadas às empresas que participavam na formação do cartel na RMSP foram efetivas, visto que, no período de 2002 a 2013, não há indícios de que um cartel operasse nesse mercado.
\end{abstract}

Palavras-chave: Poder de mercado. NEIO. Pedra britada. RMSP.

Abstract: The objective of this study is to analyze the conduct of companies of crushed stone industry in the Metropolitan Region of São Paulo (MRSP), Brazil. The methodological approach is based on the New Empirical Industrial Organization (NEIO) and refers to the market power and cartel formation after the conviction of these practices. The results indicated that the industry has a small but significant market power. At the same time, the industry behavior is different from a cartel behavior. In this way, it is possible to conclude that complaints and sanctions imposed to companies that participated in the formation of the cartel in the MRSP were effective, since in the period from 2002 to 2013 there is no evidence that a cartel operates in this market.

* Os autores agradecem o apoio financeiro do Conselho Nacional de Desenvolvimento Científico e Tecnológico (CNPq), da Fundação de Amparo à Pesquisa do Estado de Minas Gerais (Fapemig) e da Coordenação de Aperfeiçoamento de Pessoal de Nível Superior (Capes).

** Doutor em Economia Aplicada pela Universidade Federal de Viçosa (UFV). Professor adjunto do Departamento de Economia Rural da UFV. E-mail: denis.cunha@ufv.br

*** Doutorando em Economia Aplicada pela Universidade Federal de Viçosa (UFV). E-mail: lucas. pinha@ufv.br

**** Doutor em Economia Aplicada pela Universidade Federal de Viçosa (UFV). Professor titular do Departamento de Economia Rural da UFV. E-mail: mjbraga@ufv.br

***** Doutor em Economia Aplicada pela Universidade Federal de Viçosa (UFV). Professor associado do Departamento de Economia Rural da UFV. E-mail: acoelho@ufv.br 
Keywords: Market power. NEIO. Crushed stone. MRSP.

JEL Classification: D43; L13.

\section{Introdução}

A pedra britada (ou brita) faz parte do segmento do setor mineral que produz matéria-prima bruta ou beneficiada (agregados) para utilização no setor da construção civil. Além de ser insumo essencial à produção de concreto, a brita também é empregada em obras de saneamento, pavimentação, lastro de ferrovias, enrocamentos e drenagem. O estado de São Paulo é o principal produtor e consumidor, concentrando, em 2013, 27\% do total nacional (BRASIL, 2016). A Região Metropolitana de São Paulo (RMSP ${ }^{1}$ ) é a maior consumidora de brita do Brasil e concentra cerca de $40 \%$ das minas que produzem rochas britadas no estado de São Paulo. Segundo Poletto (2006), o setor de pedra britada dessa região assume grande importância na medida em que a escala de produção e consumo demandam enormes quantidades do produto para seu abastecimento.

A indústria de comercialização de pedra britada na RMSP estrutura-se como um oligopólio, cujo produto é homogêneo e o mercado é regionalizado. De acordo com dados apresentados pela Secretaria de Acompanhamento Econômico (BRASIL, 2008), a concentração no mercado de comercialização de brita na RMSP é elevada. Apenas sete firmas respondem por aproximadamente 95\% do mercado, sendo que a participação das três maiores firmas representa cerca de $50 \%$ das vendas. Segundo Oliveira et al. (2008), o alto custo de distribuição (transporte) do produto configura-se como fator limitante do raio de atuação das firmas, fazendo com que a extensão da oferta não ultrapasse 75 quilômetros, sendo o comércio internacional restrito ou inexistente. Essa realidade é verificada também em outros países. Por exemplo, segundo Kecojevic et al. (2004), os preços dos agregados naturais (pedra britada, areia e cascalho) para o consumidor final nos Estados Unidos dependem fortemente dos custos de transporte. Visto que eles apresentam baixa relação entre valor e quantidade (grande quantidade deve ser transportada para que seja lucrativo), o transporte ocorre frequentemente para mercados e consumidores próximos, dando a entender que esta é uma característica intrínseca do setor e a regionalização não ocorre apenas no Brasil e na RMSP. A possibilidade de utilização de outro recurso em substituição à pedra britada, seja industrializado ou natural, é quase nula. Além disso, a entrada de empresas no setor apresenta elevadas dificuldades relacionadas aos altos investimentos iniciais e barreiras legais e, ou, regulatórias.

1 Situada no Sudeste do estado de São Paulo, a RMSP é composta por 39 municípios e corresponde a 3,24\% do território estadual. Sua população, conforme o IBGE (2014), é de aproximadamente 20 milhões de habitantes. 
A essencialidade do produto, a dificuldade de substituição, as barreiras à entrada e o alto grau de concentração são particularidades do setor que favorecem a formação de cartéis. De fato, no ano de 2002, a Secretaria de Direito Econômico (SDE) recebeu a denúncia de um suposto cartel que envolvia empresas produtoras de brita na RMSP. Após processo administrativo, no ano de 2004 a SDE concluiu que 18 empresas e o Sindicato da Indústria de Mineração de Pedra Britada do Estado de São Paulo (Sindipedras) deveriam ser condenados por prática de cartel (CONSELHO ADMINISTRATIVO DE DEFESA ECONÔMICA, 2007). Segundo o Conselho Administrativo de Defesa Econômica (BRASIL, 2009), esse caso foi um marco para o Sistema Brasileiro de Defesa da Concorrência por conter a primeira atividade de busca e apreensão em cartéis no Brasil. Além das evidências econômicas, diversas provas foram coletadas, inclusive um sofisticado software que direcionava as vendas e fiscalizava o cumprimento dos acordos.

Com o intuito de verificar os indícios de cartel do ponto de vista econômico, Oliveira et al. (2008) repetiram o exercício econométrico no qual a SDE se baseou para emitir seu parecer utilizando dados de uma das empresas condenadas. ${ }^{2} \mathrm{~A}$ análise empírica foi baseada em testes econométricos de quebra estrutural das séries de preços de pedra britada e na estimação de modelos VAR (Vector Autoregressive Model). Os resultados apresentados pelos autores indicam que não havia aceleração dos preços no período de suposta cartelização (janeiro de 1995 a dezembro de 2004) e que houve queda do mark-up da firma estudada, o que evidenciaria comportamento próximo ao de um mercado competitivo.

Levando em consideração os pareceres emitidos pelos órgãos de defesa da concorrência a favor da existência do cartel, bem como as punições aplicadas, o presente estudo tem como objetivo analisar a conduta das empresas que compõem a indústria mineradora de pedra britada da RMSP. Essa conduta se refere ao exercício do poder de mercado e à formação de cartel após a denúncia ao Ministério Público em 2002. Procurou-se, com a análise, obter melhor entendimento da estrutura de mercado do setor, em relação ao parecer emitido pela SDE e ao estudo de Oliveira et al. (2008).

Ainda que a identificação de quebras estruturais possa oferecer indícios de comportamento não competitivo, tal como em Oliveira et al. (2008), existem metodologias específicas para a análise de poder de mercado e comportamento colusivo da indústria, como é o caso dos modelos da Nova Organização Industrial Empírica. Ademais, além da análise de Oliveira et al. (2008), não há outros estudos que tratem desse tema na indústria de pedras britadas na RMSP, principalmente no que se refere ao período posterior à denúncia e condenação da prática de cartel. Como destaca Kulaif (2001), o setor é considerado um segmento atípico, com da-

2 O exercício econométrico consta no parecer do Processo Administrativo da SDE n ${ }^{o}$. 08012.002127/2002-14. 
dos estatísticos escassos e poucos trabalhos publicados. Portanto, o presente trabalho contribui com a literatura no sentido de analisar um mercado pouco estudado, para um período ainda não analisado (posterior à denúncia de cartel) e com base em metodologia específica para tal propósito.

Além desta introdução, o trabalho está organizado em outras três seções: na segunda seção, é apresentada a metodologia, contendo os modelos teórico e analítico, bem como a descrição e a fonte dos dados; na terceira seção, são discutidos os resultados; e, por fim, na quarta seção, são apresentadas as considerações finais.

\section{Metodologia}

O paradigma Estrutura-Conduta-Desempenho (ECD) foi a principal referência teórica para as pesquisas empíricas em Organização Industrial durante décadas. Conforme Martin (1993), a hipótese central do modelo é que há relação estável e causal entre a estrutura da indústria, a conduta das firmas e o desempenho do mercado. Os modelos empíricos desenvolvidos procuravam estabelecer relações entre a parcela de mercado da firma e o grau de concentração da indústria com o poder de mercado. Contudo, a partir dos trabalhos de Bresnahan (1982), Lau (1982), Bresnahan (1989) e, mais recentemente, no Brasil, os estudos de Zeidan e Resende (2009), Profeta e Braga (2011), Beiral et al. (2013) e Fernandes e Braga (2013), a abordagem conhecida como Nova Organização Industrial Empírica (New Empirical Industrial Organization - NEIO) começou a se destacar na literatura como modelo de análise do poder de mercado. ${ }^{3}$

De acordo com Church e Ware (2000), a abordagem da NEIO tem algumas características peculiares, entre as quais é possível destacar: dados contábeis e relacionados aos custos não são utilizados, assumindo, portanto, que os custos marginais não são observados; o foco é a estimação do poder de mercado em uma única indústria (são analisadas as firmas ou a indústria como um todo, dependendo da metodologia), portanto são evitados pressupostos referentes à simetria entre indústrias distintas - o comportamento das firmas é baseado em estruturas teóricas de oligopólio. Segundo Levin (2010), os métodos modernos de pesquisa em Organização Industrial surgiram para lidar com dois obstáculos existentes até meados de 1970. O primeiro é a ausência de dados confiáveis, visto que as análises de poder de mercado eram feitas para firmas. Já o segundo está relacionado à ausência de modelos teóricos robustos que explicassem a competição imperfeita. $\mathrm{O}$ desenvolvimento moderno da teoria dos $\operatorname{jogos}^{4}$ permitiu que pesquisadores

3 Para uma análise detalhada sobre o paradigma ECD e as características da NEIO, ver Martin (1993), Bresnahan (1982), Lau (1982), Bresnahan (1989), Borenstein et al. (1999), Carlton e Perloff (2005) e Lee (2007).

4 Essa afirmação refere-se ao desenvolvimento moderno, a partir da década de 1970, denotado 
modelassem estruturas imperfeitas de concorrência, incorporando ao campo da Organização Industrial questões relacionadas à interação estratégica, equilíbrios de Nash, modelos estáticos e dinâmicos, entre outros.

Nas análises empíricas da NEIO são utilizados dados de preço e quantidade de equilíbrio de mercado para a elaboração de modelos econométricos estruturais, baseados na teoria microeconômica de maximização de lucros em ambiente de interação estratégica, visto que as decisões de uma firma se refletem nas demais. Trata-se de fazer inferências a respeito de como as firmas se comportariam em estruturas de mercado distintas, de modo que o grau de poder de mercado seja identificado com base na conduta das firmas (LEE, 2007). Mais especificamente, parte-se de uma função de demanda e de uma função de custo marginal e, pela igualdade entre receita marginal e custo marginal (condição de maximização de lucro de primeira ordem), obtém-se a relação de oferta e, posteriormente, o grau de poder de mercado.

O modelo apresentado a seguir, que fundamenta teoricamente este trabalho, é uma adaptação de Bresnahan (1982), baseado em Deodhar e Sheldon (1995). ${ }^{5}$ Outros estudos que utilizaram essa metodologia foram os de Hatirli et al. (2003), Hatirli (2004) e Deodhar e Pandey (2006).

\subsection{Modelo Teórico}

O modelo teórico tem como base o equilíbrio de Cournot-Nash com $n$ firmas, tal como apresentado em Martin (2010). Ao considerar a variação conjectural, isto é, a reação de uma determinada firma quando as demais alteram a quantidade produzida, é possível obter um parâmetro de comportamento da indústria que indica a existência de concorrência perfeita, equilíbrio de Cournot-Nash ou cartel.

Em uma indústria na qual $\mathrm{n}$ firmas produzem um produto homogêneo $\left(q_{1}, q_{2}, \ldots, q_{n}\right)$, a demanda de mercado pode ser representada pela função implícita: $Q=q(p, Z)$, em que $Q$ é a quantidade total demandada, $p$ é o preço do produto e $Z$ é o vetor de variáveis exógenas que afetam a demanda, como a renda e o preço de bens substitutos e, ou, complementares. Por sua vez, o custo marginal $(C M g)$ agregado da indústria é dado por $C M g=c(Q, W)$, em que $W$ é um vetor de variáveis exógenas do lado da oferta que deslocam a função custo, como, por exemplo, o preço dos insumos.

por Tirole (1988) de "New Theoretical Industrial Organization". A interface entre teoria dos jogos, estrutura de mercado e interações estratégicas é antiga, vide os modelos de Cournot e Bertrand do século XIX e Von Neumann e Morgenstern (1944).

5 Salienta-se que foram feitas tentativas de estimar o modelo de Steen e Salvanes (1999), bem como a modelagem alternativa, proposta por Hjalmarsson (2000). Todavia, em ambas alternativas os modelos estimados não atenderam ao pressuposto de ausência de separabilidade da função de demanda em um vetor de variáveis exógenas, não sendo possível a identificação do poder de mercado por meio da modelagem dinâmica. 
Definindo a receita total (RT) como $R T=p Q=p(Q, Z) Q$, a condição de primeira ordem implica que $\mathrm{RMg}=\mathrm{CMg}$ e pode ser escrita como:

$$
R M g(\lambda)=p+\lambda Q\left[\frac{\partial p}{\partial Q}\right]=C M g(Q, W)
$$

em que $\lambda$, chamado de parâmetro comportamental, representa a derivada da quantidade total ofertada com relação à quantidade ofertada por uma determinada firma i.

O parâmetro comportamental representa a capacidade de a indústria adotar um preço superior ao custo marginal. Assim, se $\lambda=0$, a indústria tem o comportamento de concorrência perfeita ( $p=C M g$ ), ao passo que $\lambda=1$ refere-se ao "cartel perfeito" ou monopólio; valores intermediários do parâmetro estão relacionados com soluções de oligopólio, como, por exemplo, $\lambda=1 / n$, que caracteriza o equilíbrio de Cournot-Nash.

Supondo que as $\mathrm{n}$ firmas sejam simétricas, ou seja, possuam estruturas de custos idênticas e ofertem o mesmo montante de produto, a equação (1) pode ser generalizada para o mercado como um todo e reescrita por:

$$
p(Q)+\frac{\partial p}{\partial Q}\left[\frac{1+(n-1) v}{n}\right] Q=C M g
$$

em que $v$ é a variação conjectural da firma i em relação a cada uma de suas rivais. Segundo Martin (1993), esse termo é utilizado para descrever a mudança percentual no produto de todas as demais firmas do mercado, que a empresa i espera que ocorra, em resposta à variação em sua própria produção. $\mathrm{O}$ valor de $\mathrm{v}$ seria igual a $-1 /(n-1), 1$ ou 0 para os cenários de concorrência perfeita, monopólio e Cournot-Nash, respectivamente. Nesse caso, o parâmetro $\lambda$ é definido por $\lambda=\left[\frac{1+(n-1) v}{n}\right]$.

Nota-se, portanto, que a $R M g$ é a peça fundamental para compreender o possível poder de mercado exercido pela indústria. Dela origina-se a variação conjectural $(v)$ e consequentemente o parâmetro comportamental $(\lambda)$, sendo este o principal termo a ser analisado.

\subsection{Modelo Analíitico}

A análise do poder de mercado pode ser dividida em duas etapas, conforme o modelo teórico descrito anteriormente. Na primeira estimam-se a função de demanda e a relação de oferta, ao passo que na segunda é calculado o parâmetro $\lambda$ a partir dos coeficientes estimados. A especificação e estimação da função de demanda e da relação de oferta é a estratégia empírica de obter o parâmetro de 
interesse com base no modelo teórico. O desenvolvimento econométrico das etapas é apresentado a seguir.

A regressão estimada referente à função de demanda é expressa por:

$$
Q_{t}=\beta_{0}+\beta_{1} p_{t}+\beta_{2} C C_{t}+\beta_{3} Q_{t-1}+\varepsilon_{t}
$$

em que: $Q_{t}$ é a quantidade de pedra britada vendida na RMSP; $p_{t}$ é o preço de pedra britada; $C C_{t}$ é o consumo de cimento, que é um bem complementar e funciona como um deslocador da demanda (conforme exposto na equação (1)); $Q_{t-1}$ é a quantidade de pedra britada vendida na RMSP defasada um período; $\beta_{0}, \ldots, \beta_{3}$ são os parâmetros a serem estimados; $\varepsilon_{t}$ é o termo de erro aleatório.

Assim como em Deodhar e Sheldon (1995), supõe-se que os custos marginais sejam constantes com relação ao produto e, dessa forma, são evitados problemas de identificação no modelo. Considerando que a indústria apresenta elevados custos fixos referentes à extração, manipulação e transporte, pressupor uma função de custo total contendo uma parcela de custos fixos e uma parcela linear de custos variáveis resulta em custos variáveis decrescentes e custos marginais constantes com relação ao produto, o que é uma pressuposição razoável. Em adição, se os custos marginais não fossem constantes com relação ao produto, haveria um problema de identificação inicial, visto que a quantidade afetaria $C M g$ e $R M g$ simultaneamente. Com essa pressuposição há apenas a simultaneidade entre a equação de demanda e a relação de oferta, que pode ser tratada com métodos de variáveis instrumentais. O custo marginal agregado é definido em função do preço dos principais insumos utilizados na produção de pedra britada:

$$
C M g_{t}=\alpha_{0}+\alpha_{1} W_{t}
$$

em que $W_{t}$ representa as variáveis que deslocam a função de custos, como os gastos com mão de obra, insumos e despesas de manutenção.

Considerando a equação (4) e a função de custo marginal definida na seção anterior, obtém-se o que pode ser chamado de relação de oferta: ${ }^{6}$

$$
p_{t}=\alpha_{0}+\alpha_{1} P O_{t}+\alpha_{2} Q_{t}+\alpha_{3} p_{t-1}+\mu_{t}
$$

em que $\alpha_{0}, \alpha_{1}, \alpha_{2}$ e $\alpha_{3}$ são os parâmetros e $\mu_{t}$ o termo de erro aleatório. As variáveis exógenas que deslocam a relação de oferta são: $P O_{t}$, preço do óleo diesel no estado de São Paulo; $\mathrm{p}_{\mathrm{t}-1}$, preço da pedra britada defasado um período.

6 As relações de oferta não são curvas de oferta no sentido estrito da Teoria Econômica. Segundo Martin (2001), curvas de oferta somente são definidas em mercados competitivos, dado que elas assumem independência da decisão dos agentes econômicos. 
A partir da função de demanda e da relação de oferta, utiliza-se a especificação de $\alpha_{2}$ para obter o valor do parâmetro $\lambda$. Ao derivar a equação (3) com relação a $Q_{t}$ obtém-se $\frac{\partial p_{t}}{\partial Q_{t}}=\frac{1}{\beta_{1}}$. Por sua vez, $\alpha_{2}=-\lambda \frac{\partial p_{t}}{\partial Q_{t}}$. Segue-se, portanto,
que $\lambda=-\beta_{1} \alpha_{2}$.

Deve-se atentar que a modelagem econométrica da equação de demanda e relação de oferta envolve a especificação de um modelo com variáveis endógenas que também participam como explicativas (quantidade e preço). Nesse caso, a aplicação do método de Mínimos Quadrados Ordinários (MQO) em cada equação individualmente resultaria em estimativas inconsistentes, a menos que se possa provar que $p_{t}$, na equação (3), se distribui independentemente de $\varepsilon_{t}$ e que $Q_{t}$, em (5), se distribui independentemente de $\mu_{t}$. Se houver simultaneidade, uma alternativa para obter estimadores consistentes e eficientes é utilizar o método de Mínimos Quadrados em Dois Estágios (MQ2E) (JUDGE et al.,1988). Todavia, no caso de haver correlação contemporânea entre os termos de erro das equações, o mais indicado é estimar ambas como um sistema, em que tanto a endogeneidade quanto a possível correlação entre os erros são consideradas. Tem-se, nesse caso, uma estimação por Mínimos Quadrados de Três Estágios (MQ3E). O MQ3E pode ser entendido como uma junção do MQ2E com o Seemingly Unrelated Regressions (SUR), em que cada equação é estimada isoladamente levando em consideração a endogeneidade e, posteriormente, o sistema é estimado como um todo considerando as possíveis correlações entre os erros. ${ }^{7}$

O MQ3E é utilizado nas estimações do presente trabalho, juntamente com os testes de Hansen-Sargan e o teste de Breusch-Pagan de correlação contemporânea. O primeiro é equivalente ao teste de Sargan e apresenta a hipótese nula de que os instrumentos utilizados são válidos, ao passo que o segundo indica que os erros das equações são contemporaneamente correlacionados, e por isso é justificada a utilização do MQ3E.

\subsection{Variáveis e Fonte dos Dados Utilizados}

Conforme a especificação da função de demanda e da relação de oferta no modelo analítico, as fontes e descrições das variáveis estão apresentadas na Tabela 1. As duas primeiras variáveis são endógenas e estão presentes na demanda e na oferta. As duas seguintes são as variáveis exógenas (ou pré-determinadas) da função de demanda, ao passo que as duas últimas são as variáveis exógenas (ou pré-determinadas) da relação de oferta.

7 Está além do escopo deste estudo descrever cada um desses modelos econométricos. Mais detalhes podem ser obtidos em Greene (2008). 
Tabela 1 - Descrição e fonte das variáveis utilizadas

\begin{tabular}{|c|c|}
\hline Sigla & Descrição/Fonte \\
\hline$Q_{t}$ & $\begin{array}{l}\text { Quantidade (em toneladas) de brita comercializado } \\
\text { na RMSP, obtida por meio do Sindicato da Indústria } \\
\text { de Mineração de Pedra Britada do Estado de São } \\
\text { Paulo (2014). }\end{array}$ \\
\hline$p_{t}$ & $\begin{array}{l}\text { Preço (R } \$ / \text { toneladas) da pedra britada. A série é dis- } \\
\text { ponibilizada pela Construdata (2014a) e deflaciona- } \\
\text { da pelo INCC-DI (Índice Nacional da Construção Ci- } \\
\text { vil - Disponibilidade Interna), índice elaborado pela } \\
\text { Fundação Getúlio Vargas e obtido em Construdata } \\
(2014 b) \text {. }\end{array}$ \\
\hline$Q_{t-1}$ & Quantidade de brita defasada um período. \\
\hline$C C_{t}$ & $\begin{array}{l}\text { Consumo de cimento (em mil toneladas) na cida- } \\
\text { de de São Paulo, disponibilizado pelo Construdata } \\
\text { (2014c). Esta variável representa a variação no con- } \\
\text { sumo de um bem complementar à pedra britada. }\end{array}$ \\
\hline$p_{t-1}$ & Preço da brita defasado um período. \\
\hline $\mathrm{PO}_{t}$ & $\begin{array}{l}\text { Preço do óleo diesel no estado de São Paulo (em } \\
\text { R\$/litro), disponibilizado pela Agência Nacional do } \\
\text { Petróleo, Gás Natural e Biocombustíveis (BRASIL, } \\
2014 a \text { ) e deflacionado pelo IGP-DI (Índice Geral de } \\
\text { Preços - Disponibilidade Interna). Representa os cus- } \\
\text { tos do maquinário envolvido no processo de brita- } \\
\text { gem e de transportes. }\end{array}$ \\
\hline
\end{tabular}

Fonte: Elaboração própria.

De acordo com as etapas do processo produtivo de pedra britada descritas no estudo de Oliveira et al. (2008), os gastos com o óleo diesel representam um dos principais custos relacionados à produção. Outros dois importantes componentes do custo são os gastos com explosivos e manutenção das máquinas utilizadas no processo produtivo. Todavia, não foram obtidos dados estatísticos que pudessem representar tais despesas. Vale ressaltar que uma dummy referente à crise econômica a partir de setembro de 2008 foi testada nas estimações, porém esta não se mostrou relevante para os resultados.

No MQ3E, os instrumentos a serem utilizados são os mesmos para ambas as equações. Dessa forma, os instrumentos foram todas as variáveis exógenas e pré-determinadas do sistema, além de uma tendência determinística.

Em todas as variáveis, o subscrito $t$ indica o período de tempo (de fevereiro de 2002 a dezembro de 2013). Portanto, pretende-se analisar a conduta das empresas após a denúncia de cartel, com o intuito de verificar, econometricamente, 
se o mercado passou a ter características de concorrência perfeita, Cournot-Nash ou cartel. As estatísticas descritivas das variáveis, assim como a evolução do preço e da quantidade de pedra britada comercializada na RMSP no período considerado na pesquisa, são apresentadas no Anexo.

\section{Resultados e Discussão}

A equação de demanda e a relação de oferta foram estimadas como um sistema por $\mathrm{MQ} 3 \mathrm{E},{ }^{8}$ em que as variáveis exógenas e pré-determinadas são utilizadas como instrumentos, além da tendência. O teste de Hansen-Sargan ${ }^{9}$ apresentou valor Qui-quadrado de 7,29 com p-valor de 0,12, indicando que os instrumentos utilizados são válidos a 1\% de significância. Já o teste de Breusch-Pagan de correlação contemporânea ${ }^{10}$ apresentou a estatística ML (Multiplicador de Lagrange) de 3,63 com p-valor de 0,057, sugerindo que os erros são correlacionados a $10 \%$ de significância.

A Tabela 2 contém as estimativas da função de demanda. $\mathrm{O} \mathrm{R}^{2}$ apresentou valor de $85 \%$, o que indica bom ajuste do modelo. Todos os coeficientes mostraram-se significativos a $1 \%$ de significância estatística, ao mesmo tempo em que os sinais estão de acordo com o esperado. O aumento de $10 \%$ no preço da pedra britada, tudo o mais mantido constante, reduziria a quantidade demandada na RMSP em, aproximadamente, 3,3\%; da mesma forma, o acréscimo de $10 \%$ no consumo de cimento levaria ao aumento de $6,3 \%$ no consumo de brita, o que confirma a complementaridade entre brita e cimento, utilizados em conjunto na produção de concreto. Nota-se também a relevância da quantidade defasada na explicação da quantidade no período atual.

8 Para a estimação do modelo foi utilizado o comando "reg3" do software STATA 12.0 (StataCorp LP, College Station, TX, EUA).

9 A hipótese nula do teste é de que os instrumentos são válidos. O comando para o teste no STATA 12.0 é o "overid", desenvolvido por Baum et al. (1999).

10 A hipótese nula do teste é de que os erros não são correlacionados. O comando para o teste no STATA 12.0 é o "Imcovreg3", desenvolvido por Shehata (2012). 
Tabela 2 - Parâmetros estimados da função de demanda

\begin{tabular}{cccc}
\hline Variável & Coeficiente & Erro padrão & P-valor \\
\hline Intercepto & $3,52^{* * *}$ & $(0,54)$ & 0,000 \\
$p_{t}$ & $-0,33^{* * *}$ & $(0,08)$ & 0,000 \\
$C C_{t}$ & $0,63^{* * *}$ & $(0,07)$ & 0,000 \\
$Q_{t-1}$ & $0,53^{* * *}$ & $(0,05)$ & 0,000 \\
\hline Instrumentos & Variáveis exógenas do sistema e tendência \\
$\mathrm{R}^{2}$ & $0,85 \%$ & \\
\hline
\end{tabular}

Fonte: Elaboração própria a partir dos resultados da pesquisa.

Nota: Variáveis em logaritmo. (***) indica significância a $1 \%$.

A baixa elasticidade-preço da demanda está de acordo com a expectativa, visto que, além de ser um insumo essencial à construção civil, a possibilidade de substituição de brita é bastante reduzida (o cascalho e as escórias siderúrgicas são os poucos exemplos de produtos substitutos). Como argumentaram Schmidt e Lima (2006) e Zeidan e Resende (2009), que também analisaram mercados de produtos com reduzida possibilidade de substituição (aço e cimento, respectivamente), a baixa elasticidade aumentaria a probabilidade de exercício do poder de mercado pelas firmas do setor, como de fato ocorreu na RMSP antes da denúncia ter sido realizada. Esse fato é agravado devido à dificuldade de importar o produto de outras regiões, já que, para distâncias maiores que aproximadamente 75 quilômetros, o preço da brita é consideravelmente majorado pelo custo do frete.

A relação de oferta estimada está apresentada na Tabela 3. A variável endógena é a quantidade de pedra britada $\left(Q_{t}\right)$ e o modelo estimado está adequadamente ajustado de acordo com o $\mathrm{R}^{2}$ de $99 \%$. Todos os coeficientes apresentaram o sinal esperado e foram estatisticamente significativas a 1\% de significância. Pelos resultados, pode-se afirmar que, se houver acréscimo de $10 \%$ no preço do litro do óleo diesel, tudo o mais mantido constante, seria necessário um aumento de aproximadamente $0,6 \%$ no preço da brita para que as pedreiras estivessem dispostas a manter sua produção constante. O sinal positivo da variável $Q_{t}$ indica que as firmas somente aumentariam sua quantidade ofertada de brita se houvesse um reajuste positivo no preço. 
Tabela 3 - Parâmetros estimados da relação de oferta

\begin{tabular}{cccc}
\hline Variável & Coeficiente & Erro padrão & P-valor \\
\hline Intercepto & $-0,35 * * *$ & $(0,10)$ & 0.000 \\
$\mathrm{Q}_{\mathrm{t}}$ & $0,03^{* * *}$ & $(0,01)$ & 0,000 \\
$\mathrm{p}_{\mathrm{t}-1}$ & $0,99 * * *$ & $(0,01)$ & 0,000 \\
$\mathrm{PO}_{\mathrm{t}}$ & $0,06^{* * *}$ & $(0,01)$ & 0,000 \\
\hline Instrumentos & Variáveis exógenas do sistema e tendência \\
$\mathrm{R}^{2}$ & $0,99 \%$ & \\
\hline
\end{tabular}

Fonte: Elaboração própria a partir dos resultados da pesquisa.

Nota: Variáveis em logaritmo. (**) indica significância a $1 \%$.

O valor do coeficiente estimado para a variável $Q_{t}$ indica que a oferta é sensível a aumentos no preço, o que pode ser explicado pela possibilidade de mudança na quantidade produzida em curto período de tempo. É possível inferir que a produção de brita está diretamente relacionada com a capacidade de produção horária de seus equipamentos. Assim, para aumentar a produção, é necessário que se obtenha melhor desempenho da configuração e da estrutura atual. A firma pode facilmente dobrar as horas trabalhadas e, dessa forma, dobrar sua produção. Porém, se a empresa já tiver a escala de horas completa e não possuir dias livres para produção, a ampliação estrutural será inevitável. Embora a compra de novos equipamentos, além de dispendiosa, possa demorar, existe a possibilidade do aluguel, que é mais rápido e pode ser utilizado apenas em períodos de grande demanda.

Finalmente, a partir das estimativas da demanda e da relação de oferta, pôde-se obter o parâmetro que representa o grau de poder de mercado. Conforme especificado no modelo analítico, $\lambda=-\beta_{1} \alpha_{2}$. Como $\beta_{1}=-0,33$ e $\alpha_{2}=0,03$, tem-se que $\lambda \cong 0,01$. Assim como a expectativa teórica, $\lambda$ está compreendido no intervalo entre 0 e 1 e o valor obtido sugere que a indústria de mineração de pedra britada da RMSP está distante da situação de cartel.

Com o intuito de garantir a robustez dos resultados, foi realizado o procedimento de bootstrap nas estimações. Os resíduos da relação de oferta e da equação de demanda foram reamostrados 1.000 vezes e incorporados às variáveis endógenas, gerando novas séries de quantidade e preço da pedra britada em cada etapa. Em seguida, o MQ3E foi aplicado. Assim, para cada etapa, estimou-se um valor de $\alpha_{2}, \beta_{1}$ e, consequentemente, $\lambda$. Com base nas 1.000 estimativas de $\lambda$, a média calculada foi de 0,0098 , com desvio padrão de 0,0028 (valores máximos e mínimos iguais a 0,0208 e 0,0027 , respectivamente). Como o desvio padrão foi muito baixo, os intervalos de confianças estimados foram pequenos (limites inferior e superior 
de 0,0097 e 0,01 aproximadamente), indicando que o parâmetro $\lambda \cong 0,01$ é um resultado bastante robusto.

Testes de hipóteses foram realizados para verificar se o parâmetro comportamental é estatisticamente igual às estruturas de concorrência perfeita, Cournot-Nash ou cartel perfeito. No primeiro caso a hipótese nula é que $\lambda=0$, no segundo caso $\lambda=1 / 18 \cong 0,05$ (considerando que a indústria era composta pelas 18 firmas condenadas pelo cartel) e no terceiro caso $\lambda=1$. Em todos os casos a hipótese nula foi rejeitada. Portanto, a partir desses resultados, pode-se afirmar que a indústria apresenta poder de mercado baixo, porém significativo. Isso leva a dois importantes resultados principais, quais sejam, a indústria de pedra britada da RMSP se afasta da situação de concorrência perfeita e, ao mesmo tempo, está distante do padrão comportamental característico de cartel.

Com relação ao primeiro resultado, há, na literatura, argumentos favoráveis ao exercício de poder de mercado no mercado de pedra britada. O exercício e a manutenção do poder de mercado são facilitados pela existência de barreiras à entrada. Como argumentaram Neves e Silva (2007), o desenvolvimento de uma área até o início da exploração requer grande capacidade financeira própria, ou acesso a linhas de financiamento, devido à presença de custos irrecuperáveis. A extração de brita é uma atividade capital-intensiva e os custos fixos envolvidos são elevados. Ou seja, há significativa barreira à entrada resultante do alto investimento inicial que deve ser realizado para adquirir uma pedreira e a maquinaria. Há ainda barreiras legais ou regulatórias. Conforme Oliveira et al. (2008), para a exploração das minas é preciso obter um título minerário sobre as jazidas junto ao Ministério de Minas e Energia e parecer favorável no relatório de impacto ambiental realizado pela Secretaria do Meio Ambiente. Segundo estudo do DNPM (2002), além de representarem significativos custos, as referidas licenças levam muito tempo para serem expedidas. Além das barreiras à entrada, outros fatores que favorecem o exercício do poder de mercado são a regionalização do comércio, a baixa relação entre valor e quantidade, a essencialidade do produto e a dificuldade de substituição, características que já haviam sido discutidas na introdução deste estudo.

Já com relação ao segundo resultado, levando em consideração a decisão judicial de que realmente houve a formação de cartel no final da década de 1990, o presente trabalho verificou que, de acordo com a metodologia empregada, após o ano de 2002, o mercado de pedra britada na RMSP está distante do comportamento de um cartel perfeito. Isso pode sugerir que as punições sofridas devido à condenação surtiram efeito, indicando que as práticas anticompetitivas têm diminuído consideravelmente no período analisado. 


\section{Considerações Finais}

Os resultados do presente estudo permitem concluir que a indústria de pedra britada na RMSP apresenta pequeno e significativo poder de mercado, suficiente para distanciá-la da estrutura de concorrência perfeita e, ao mesmo tempo, do comportamento de cartel. Conclui-se, desta forma, que as investigações e as punições sofridas pelas 18 firmas condenadas têm se mostrado efetivas no sentido de reduzir as práticas anticompetitivas que estavam sendo praticadas. Vale ressaltar que restringir práticas anticoncorrenciais neste setor é de fundamental importância, visto que a possível formação de cartel poderia ser prejudicial à sociedade como um todo, principalmente quando se considera o aquecimento do setor de construção civil no período analisado. Além disso, o Brasil apresenta grande demanda reprimida por agregados, devido ao histórico déficit habitacional, o que poderia aumentar a ineficiência gerada pela prática de preços acima do custo marginal.

Finalmente, o estudo da indústria de pedras britadas permitiu uma aplicação mais favorável da metodologia da NEIO, já que se trata efetivamente de um produto homogêneo e cujo mercado pode ser adequadamente delimitado. Há evidências consideráveis, no contexto do Sistema Brasileiro de Defesa da Concorrência, de que o mercado relevante é regional e que a RMSP forma um mercado distinto. A dificuldade em considerar mudanças estruturais, como tecnologia, foi superada ao utilizar um período de tempo relativamente curto, no qual é possível afirmar que não houve mudanças tecnológicas significativas no setor. No entanto, a metodologia empregada é conhecida na literatura como uma análise estática, e por isso é sugerido para futuros estudos que métodos dinâmicos de estimação de poder de mercado sejam utilizados.

\section{Referências}

BAUM, C. F. et al. Overid: tests of overidentifying restrictions for a regression estimated via instrumental variables. Stata 12.0 command, 1999.

BEIRAL, B. R. S.; MORAES, M. A. F. D.; BACCHI, M. R. P. Concentração e poder de mercado na distribuição de etanol combustível: análise sob a ótica da nova organização industrial empírica. Economia Aplicada, v. 17, n. 2, p. 251-274, 2013.

BORENSTEIN, S.; BUSHNELL, J.; KNITTEL, C. R. Market power in electricity markets: beyond concentration measures. Energy Journal, v. 20, n. 4, p. 65- 88, 1999.

BRASIL. Ministério da Fazenda. Secretaria de Acompanhamento Econômico. Parecer técnico no. 06551/2008/RJ. Brasília, DF, 2008. Disponível em: < http://www1.seae.fazenda.gov. br/littera/pdf/08012008939200851.pdf>. Acesso em: 10 set. 2014. 
BRASIL. Ministério da Justiça. Secretaria de Direito Econômico. Conselho Administrativo de Defesa Econômica. Combate a cartéis e programas de leniência. Brasília, DF: MJ, 2009. Disponível em: < http://www.cade.gov.br/upload/Cartilha\%20Leniencia\%20SDE_CADE.pdf>. Acesso em: 15 out. 2014.

. Ministério de Minas de Energia. Agência Nacional do Petróleo, Gás Natural e Biocombustíveis. Levantamento de preços. 2014a. Disponível em: < http://www.anp.gov.br/ preco/prc/Resumo_Mensal_Index.asp> Acesso em: 20 set. 2014.

- Ministério de Minas de Energia. Departamento Nacional de Produção Mineral. Sumário Mineral 2002. Disponível em: <http://www.dnpm.gov.br/mostra_arquivo. asp?IDBancoArquivoArquivo=352 > . Acesso em: 12 set. $2014 \mathrm{~b}$.

Ministério de Minas de Energia. Departamento Nacional de Produção Mineral. Sumário Mineral 2014. 2014b. Disponível em: < http:/www.dnpm.gov.br/dnpm/sumarios/ sumario-mineral-2014>. Acesso em: 08 mar. 2016.

BRESNAHAN, T. F. Empirical studies of industries with market power. In: SCHMALENSEE, R.; WILLING, R. (Org.). Handbook of Industrial Organization. Amsterdam: North-Holland, 1989. 2. v. p. 1011-1057. 1982.

. The oligopoly solution concept is identified. Economic Letters, v. 10, n. 1-2, p. 87-92,

CARLTON, D. W.; PERLOFF, J. M. Modern industrial organization. 4. ed. Upper Saddle River: Pearson, 2005. 822 p.

CHURCH, J. R.; WARE, R. Industrial organization: a strategic approach. New York: McGrawHill, 2000.

CONSELHO ADMINISTRATIVO DE DEFESA ECONÔMICA. Guia prático do CADE: a defesa da concorrência no Brasil. 3. ed. São Paulo: CIEE, 2007. 112p.

CONSTRUDATA. Sinduscon-SP: o sindicato da construção. 2014a. Disponível em: < http:// www.construdata.com.br/packages/data/pages/load_series.tpl.php?level1[] =40Elevel2[] =7 1 Elevel3[] = 406Elevel4[] =333 > . Acesso em: 19 set. 2014.

. Sinduscon-SP: o sindicato da construção, 2014b. Disponível em: <http://www.construdata.com.br/packages/data/pages/load_series.tpl.php?level1 [] $=40 \mathcal{E}$ level2[] $=55$ Elev el3[] $=305>$. Acesso em: 19 set. 2014.

Sinduscon-SP: o sindicato da construção, 2014c. Disponível em: < http://www.construdata.com.br/packages/data/pages/load_series.tpl.php?level1 [] $=95$ \&level2[] $=96 \mathcal{E}$ lev el3[]=268> . Acesso em: 19 set. 2014.

DEODHAR, S. Y.; PANDEY, V. Degree of instant competition: estimation of market power in india's instant coffee market. Índia: Indian Institute of Management Ahmedabab, Oct. 2006. (Working Paper, n. 2006-10-02). Disponível em: <http:/www.iimahd.ernet.in/publications/ data/2006-10-02_sdeodhar.pdf>. Acesso em: 10 set. 2014. 
DEODHAR, S.Y.; SHELDON, I. M. Is foreign trade (im)perfectly competitive? An analysis of the German market for banana imports. Journal of Agricultural Economics, v. 46, n. 3, p. 336-348, 1995.

FERNANDES, F. A. S.; BRAGA, M. J. Poder de mercado no segmento de distribuição de gasolina c: uma análise a partir da nova organização industrial empírica. Estudos Econômicos, v. 43, n. 3, p. 465-497, 2013.

GREENE, W. H. Econometric analysis. 6. ed. New Jersey: Pearson Prentice Hall; Upper Saddle River, 2008. 1178 p.

HATIRLI, S. A. Is the fluid milk market competitive in Turkey? Turkish Journal of Veterinary and Animal Science, v. 28, n. 4, p. 633-668, 2004.

HATIRLI, S. A.; JONES, E.; AKTAS, A. R. Measuring the market power of the banana import market in Turkey. Turkish Journal of Agriculture and Forestry, n. 27, p. 367-373, 2003. Disponível em: <http://journals.tubitak.gov.tr/agriculture/issues/tar-03-27-6/tar-27-6-7-0305-14. pdf > . Acesso em: 13 set. 2014.

HJALMARSSON, E. Nord pool: a power market without market power. Göteborg: Göteborg University, 2000. (Working Paper in Economics, n. 28). Disponível em: <http://swopec.hhs. se/gunwpe/papers/gunwpe0028.pdf> . Acesso em: 13 set. 2014.

IBGE. Censo 2010. Disponível em: < http://www.censo2010.ibge.gov.br/primeiros_dados_divulgados/index.php>. Acesso em: 12 set. 2014.

JUDGE, G. G. et al. Introduction the Theory and Practice of Econometrics. New York: John Wiley, 1988. $1024 \mathrm{p}$.

KECOJEVIC, V.; NELSON T.; SCHISSLER, A. An analysis of aggregate production in the United States: historical data and issues facing the industry. Minerals and Energy - Raw Materials Report, v. 19, n. 4, p. 25-33, 2004.

KULAIF, Y. Análise dos mercados de matérias-primas minerais: um estudo de caso da indústria de pedras britadas do estado de São Paulo. 2001. 144 f. Tese (Doutorado em Engenharia) Escola Politécnica, Universidade de São Paulo, São Paulo, 2001.

LAU, L. J. On identifying the degree of competitiveness from industry price and output data. Economics Letters, v. 10, n. 1-2, p. 93-99, 1982.

LEE, C. SCP, NEIO and Beyond. Malaysia: Nottingham University Business School, 2007. (Working Paper Series, v. 2007-05). Disponível em: < http://www.icsead.or.jp/7publication/ workingpp/wp2007/2007-05.pdf> . Acesso em: 21 jan. 2009.

LEVIN, L. E. J. Empirical industrial organization: a progress report. Journal of Economic Perspectives, v. 24, n. 2, p. 145-162, 2010.

MARTIN, S. Advanced industrial economics. 2. ed. New York: Wiley-Blackwell, 2001. 552 p.

. Industrial economics: economic analysis and public policy. 2. ed. New Jersey: Prentice Hall, 1993. 623 p. 
MARTIN, S. Industrial organization in context. Oxford: Oxford University Press, 2010. 991 p.

NEVES, C. A. R.; SILVA, L. R. Universo da mineração brasileira. Brasília, DF: Diretoria de Desenvolvimento e Economia Mineral, 2007. Disponível em: < http://www.dnpm.gov.br/ mostra_arquivo.asp?IDBancoArquivoArquivo=2102 > . Acesso em 20 set. 2014.

OLIVEIRA, A. V. M. et al. Avaliação de cartéis: o caso das pedras britadas. Revista de Direito da Concorrência, v. 18, p. 93-121, 2008.

POLETTO, C. A exploração de pedreiras na região metropolitana de São Paulo no contexto do planejamento e gestão do território. 2006. 236 f. Tese (Doutorado em Geografia) - Faculdade de Filosofia, Letras e Ciências Humanas, Universidade de São Paulo, São Paulo, 2006.

PROFETA, G. A.; BRAGA, M. J. Poder de mercado na indústria brasileira de fertilizantes NPK (04-14-08), no período de 1993-2006. Revista de Economia e Sociologia Rural, v. 49, n. 04, p. 837-856, 2011.

SCHMIDT, C. A. J.; LIMA, M. A. M. A perda de peso morto e a elasticidade-preço da demanda do setor siderúrgico no Brasil. Estudos Econômicos, v. 36, n. 1, p. 127-147, 2006.

SHEHATA, E. A. E. LMCOVREG3: Stata module to compute breusch-pagan lagrange multiplier diagonal covariance matrix test after (3SLS-SURE) regressions. Boston: Boston College Department of Economics, 2012. Statistical Software Components.

SINDICATO DA INDÚSTRIA DE MINERAÇÃO DE PEDRA BRITADA DO ESTADO DE SÃO PAULO. Mercado de Brita: RMSP. Disponível em: < http://www.fiesp.com.br/sindipedras/ sobre-o-sindipedras/estatisticas/> . Acesso em: 10 set. 2014.

STEEN, F.; SALVANES, K. G. Testing for market power using a dynamic oligopoly model. International Journal of Industrial Organization, v. 17, n. 2, p. 147-177, 1999.

TIROLE, J. The theory of industrial organization. Cambridge, MA: MIT Press, 1988. 479 p.

VON NEUMANN, J.; MORGENSTERN, O. The theory of games and economic behavior. Princeton: Princeton University Press, 1944.

ZEIDAN, R. M.; RESENDE, M. Measuring market conduct in the Brazilian cement industry: a dynamic econometric investigation. Review of Industrial Organization, v. 34, n. 3, p. 231-244, 2009. 


\section{Anexo A}

Tabela 4 - Estatísticas descritivas das variáveis, em logaritmo

\begin{tabular}{ccccc}
\hline Variável & Média & Desvio-Padrão & Mínimo & Máximo \\
\hline$Q_{t}$ & 14,74 & 0,21 & 14,29 & 15,27 \\
$P_{t}$ & 2,78 & 0,14 & 2,53 & 2,97 \\
$C C_{t}$ & 6,91 & 0,21 & 6,47 & 7,31 \\
$P O_{t}$ & 0,03 & 0,09 & $-0,16$ & 0,19 \\
\hline
\end{tabular}

Fonte: Elaboração própria a partir dos resultados da pesquisa.

Gráfico 1 - Evolução do preço e da quantidade de pedra britada na RMSP no período de fevereiro de 2002 a dezembro de 2013

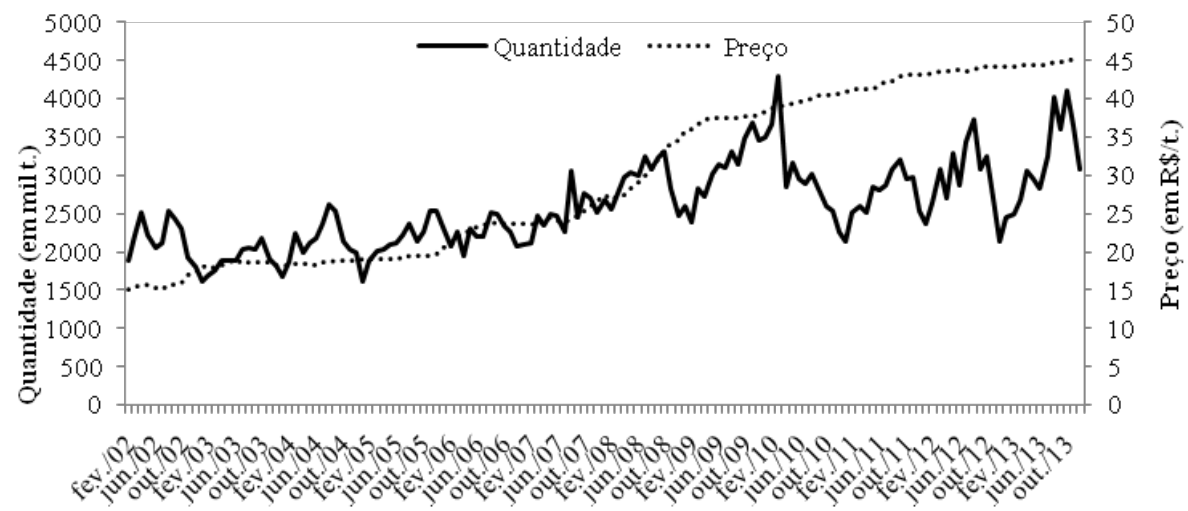

Fonte: Elaboração própria a partir de Sindicato da Indústria de Mineração de Pedra Britada do Estado de São Paulo (2014) e Construdata (2014a).

Recebido em: 09/03/2015. Aceito em: 25/04/2016. 